CHAPITRE

\title{
3 \\ La production des cadres et leur devenir : filières \\ techniques et filières générales
}

Présentée - parfois dramatiquement - comme un véritable enjeu culturel, voire comme un (non) choix de société, la " désaffection pour les études scientifiques » est perçue de façon d'autant plus cruciale que se multiplient commentaires et analyses sur les besoins à venir de cadres et techniciens scientifiques, dans un contexte de concurrence mondiale exacerbée et de brain drain nord-américain qui s'accélèrerait ${ }^{57}$.

Différents travaux, menés notamment sous l'égide du Commissariat général du plan et du ministère de l'Emploi, se sont attachés à préciser et à chiffrer les qualifications, métiers et filières nécessaires au renouvellement et au développement économique dans les prochaines années. Au-delà de l'affirmation d'un générique manque de "diplômés scientifiques », ces travaux sont l'occasion d'insister sur la très grande diversité des besoins en question, au terme d'une période où la diversification des cursus

57. « Fuite des cerveaux » qui fait a fait l'objet d'un certain nombre de travaux (tendant à la relativiser), voir notamment ceux de Philippe Moguerou (IREDU) ou encore certaines enquêtes de l'APEC, mais aussi de commentaires dans la presse oscillant entre des exemples très alarmants et un relativisme beaucoup plus mesuré sur la position française (cf. par exemple le dossier sur " La défaite de la science française " par La Recherche, avril 2002, pp. 60-73). On rappellera, par ailleurs, que si les Etats-Unis accueillent plus de 500.000 étudiants étrangers, on en compte plus de 850000 dans les établissements de l'Union européenne; cf. par ailleurs l'annexe $n^{\circ} 21$ pour des données plus détaillées. 
propre à l'enseignement supérieur depuis deux décennies est certainement à rapprocher des mutations qu'a connues l'appareil productif depuis la fin des années 1970.

Ainsi, tout en confirmant le problème spécifiquement posé au système éducatif et de recherche pour assurer son propre renouvellement, on peut déduire de ces travaux une approche nuancée des baisses statistiques propres à certains cursus ainsi que des scenarios assez contrastés sur les parcours et les formations à développer en priorité.

\section{Les besoins de qualifications à venir}

\section{1. - Rappel sur les mutations de l'appareil productif depuis les années 1970}

Avec le premier choc pétrolier s'engage la crise du système taylorien de production de masse sur lequel s'était construite la croissance des Trente Glorieuses. S'opère, plus ou moins progressivement selon les secteurs, le passage à une société post-industrielle que certains économistes préfèrent qualifier de « société industrielle de service » $\left(\mathrm{P}\right.$. Veltz $\left.{ }^{58}\right)$. Caractérisé par une moindre standardisation et une diversification des structures d'activités, l'appareil productif se « tertiarise». En effet, les emplois de loisirs et de services aux personnes connaissent un développement sans précédent. Mais, parallèlement, une large partie des nouveaux emplois " tertiaires " relève bien en fait du secteur productif dont les fonctions amont (recherche, organisation, information), aval (commercialisation, communication) et connexes (transport, logistique) se développent au détriment des fonctions de fabrication proprement dite.

Les effets sur la structure de la population active sont très significatifs ${ }^{59}$. Outre que l'effondrement du nombre d'agriculteurs et d'artisans-commerçants engagé depuis l'immédiat aprèsguerre se confirme, se produit désormais une baisse, à la fois absolue et relative, de l'emploi ouvrier (de $35 \%$ à $25 \%$ de la population active) tandis que les " cols blancs », depuis les cadres supérieurs jusqu'aux employés deviennent majoritaires dans

58. Cf. par exemple Pierre Veltz, Des ternitoires pour apprendre et innover, Paris, éditions de l'Aube, 1994.

59. Les statistiques qui suivent sont pour l'essentiel extraits de documents INSEE : voir notamment B. Seys, "L'évcilution sociale de la population active ", INSEE Première, n434, mars 1996 ; T. Amosisé, " RGP 99 l'espace des métiers 1990-1999 », INSEE Première, $n^{\circ} 790$, juillet 2001 
la population active (au sein de laquelle ils pèsent environ $65 \%$ en 1999 , contre $45 \%$ dans les années 1970 et $15 \%$ au milieu du $\mathrm{XX}^{\mathrm{e}}$ siècle).

Parmi ces cols blancs, les cadres et les professions intermédiaires (soit plus de $30 \%$ de la population active actuelle) ont vu, dans le même temps, leur niveau de formation initiale s'élever considérablement : $90 \%$ sont titulaires du baccalauréat et les deux tiers sont diplômés de l'enseignement supérieur (contre moins de la moitié dans les années 1950) ${ }^{60}$.

Ces proportions sont encore plus fortes si l'on ne considère que les jeunes actifs, sachant que globalement toutes catégories confondues les actifs ayant achevé leur études depuis moins de cinq ans sont, en 2001, diplômés de l'enseignement supérieur à $45 \%$. Pour mémoire, ils n'étaient encore que $28 \%$ en ... $1990^{61}$, car cette élévation globale du niveau de formation s'est très fortement accélérée à partir de la fin des années 1980, sous l'effet tant de la demande sociale que du besoin quasi exponentiel de l'économie en main-d'œuvre qualifiée. Cette élévation du niveau général de formation, notamment parmi les jeunes actifs, pour lesquels le baccalauréat devient une quasi-norme (à $60 \%$ ), est ainsi allée de pair avec une assez forte diversification des cursus et des diplômes, notamment par le développement de l'enseignement supérieur professionnel technologique court et long.

Ainsi, loin de marquer une désaffection sociétale pour les sciences, la diversification des cursus, notamment via les filières et diplômes technologiques et professionnels du supérieur apparaît bien en phase avec la nature des besoins passés et futurs du système productif, même si un certain nombre de décalages dans le temps et d'effets de conjonctures ont pu entraîner des phénomènes de « file d'attente » en accordéon.

60. Cf. M-A Estrade et C. Mini, " La Hausse du niveau de formation », INSEE Première, $n^{\circ} 488$, septembre 1996.

61. Cf. E. Nauze-Fichet et M. Tomasini, « Diplôme et insertion sur le marché du travail : approches socioprofessionnelle et salariale du déclassement », Economie et statistique, $n^{\circ} 354,2002$, p.22. 


\section{2. - Une explosion des besoins de qualifications : les secteurs en croissance à l'horizon 2010}

Globalement ${ }^{62}$, on considère que les besoins d'emploi très qualifié devraient continuer à croître très fortement au cours de la décennie 2000-201.0, sous l'effet d'un double phénomène : la poursuite de la croissance de l'emploi très qualifié, d'une part, les flux particulièrement forts de départ en retraite des cadres, d'autre part. Mais au-delà de cette croissance globale, les besoins nets de recrutement (créations + remplacements) s'avèrent relativement différenciés selon les secteurs.

En ce qui concerne les créations d'emplois, elles devraient tout particulièrement concerner les cadres $(+1,2$ million, soit une croissance de $25 \%$ sur la décennie $2000-2010^{63}$ ) et les professions intermédiaire $\left(+750000\right.$ soit $\left.15 \%{ }^{64}\right)$, notamment dans :

- le secteur " tertiaire », à savoir (pour les postes qualifiés) : l'informatique, la communication, la recherche, la formation continue, la gestion et les fonctions administratives 65 ;

- le secteur industriel devant connaître une croissance moindre, notamment portée par la maintenance industrielle et les industries de procédés (agroalimentaire, pharmacie) ${ }^{66}$.

En ce qui concerne les départs en retraite (calculés à partir du poids des plus de 50 ans et de différentes hypothèses d'âge moyen de départ en retraite), ils seront à partir de 2005, par exemple :

-particulièrement forts dans les secteurs de la banque, de l'enseignement (et recherche publique) et de la fonction publique (de l'ordre de $4 \%$ par an) ainsi que, dans une moindre mesure, pour les ingénieurs de l'industrie ;

62. Qu'il s'agisse des travaux de la DARES ou de ceux de la DPD, et quelle que soit l'hypothèse macro-économique (" basse " ou " haute ") qui les sous-tende. Ces travaux ont été largement exploités par le groupe mis en place au Commissariat général du plan sur la prospective des qualifications, cf. C. Seibel et C. Afriat, Avenir des métiers, CGP, 2002, 189 p. et M. Amar et al. 2005 : le choix démographique, défi pour les professions, les branches et les territoires, CGP, 2002, 159 p. On soulignera cependant que, depuis lors, les perspectives de croissance s'avèrent moins « linéaires« que prévu...

63. Soit un quasi-doublement par rapport à la décennie 1990-2000.

64. Ces chiffres correspondent au scénarios « haut » de la DARES comme de celui de la DPD, sachant que les variations par rapport aux scénarios « bas » concernent surtout d'autres catégories.

65. L'administration publique, de même que l'enseignement, étant eux supposés rester globalement stables ou augrnenter très faiblement.

66. A noter que dans les scénarios « bas " (désormais plus vraisemblables), le secteur industriel tend à baisser, mais cette baisse giobale n'exclurait pas une croissance significative pour les cadres. 
- non négligeables dans le champ des transports, de certaines professions industrielles (mécanique et électricité), de la gestion et de la recherche privée (de l'ordre de $2 \%$ par an);

- mais plutôt faibles pour l'informatique ou la communication, par exemple.

Ainsi, certains secteurs combinent (les ordres de grandeur entre parenthèses indiquent le solde - créations + départs en retraite - sur dix ans, toutes catégories socio-professionnelles confondues $\left.{ }^{67}\right)$ :

- forte croissance et faibles départs en retraite : informatique $(250000)$, communication $(150000)$, recherche $(230000)$, ingénieurs et cadres techniques de l'industrie (50000) ;

- croissance sensible et forts départs en retraite : services aux particuliers (1 400000$)$, gestion (1 000000$)$, commerce $(700000)$, ou encore santé $(600000)$ et pour l'industrie : industrie de process $(420000)$;

- croissance faible et forts départs en retraite : administration $(500000)$, banques $(200000)$, enseignement $(580000)$; et pour l'industrie : mécanique (390000), maintenance (250000), construction $(440000)$, électricité $(80000)$.

\section{Insertion et concurrences sur le « marché des diplômés ")}

Quels que soient les scénarios envisagés, les très forts besoins de recrutement à venir entraîneront de fortes tensions pour les professions intermédiaires et surtout pour les cadres, à l'instar de ce qui s'est passé lors de la reprise économique de 1998-2001 mais amplifié par l'augmentation des départs en retraite.

\section{Pour un scénario donné68, l'ampleur de ces tensions pour- rait cependant être fonction de différentes variables :}

- la mobilité professionnelle : les besoins en cadres et professions intermédiaires étant susceptibles d'être ou non pour partie satisfaits par promotion interne (ou intersectorielle), sachant que cette variable affecte différemment les secteurs professionnels : les secteurs stratégiques à petits effectifs - comme l'informatique - ont en la matière moins de ressour-

67. Travaux de la DARES.

68. Qui dépend lui-méme du taux de croissance, des âges moyens d'entrée et de sortie de la vie active, etc. 
ces que des grandes branches - type assurance, gestion, administration -, les modes de recrutement, le poids de la formation continue, etc. jouant également un rôle ;

- la concurrence entre le recrutement de jeunes diplômés et celui de chômeurs adultes ou de femmes en reprise d'activité (mais aussi le recours à l'immigration) : a priori cette variable crée asse $\mathrm{Z}$ peu de tension pour les postes de cadres (mais a des conséquences non négligeable sur le « déclassement » à l'embauche des jeunes diplômés ${ }^{69}$ ou le reclassement des actifs diplômés récemment engagés, quand elle se combine ou non avec une politique de promotion interne).

Ainsi, selon ces variables (et les différents scénarios «macro» retenus), le besoin de recrutement de diplômés de l'enseignement supérieur $(B a c+3$ et plus, Bac +2$)$ suppose qu'ils représentent entre $40 \%$ et $50 \%$ des flux de sorties de formation initiale (soit encore, selon les scenarios retenus entre 295000 et 340000 diplômés annuels) ${ }^{70}$.

Or, on rappellera qu'en 1998 par exemple, les bac +2 et $\mathrm{Bac}+3$ et plus ont représentés $37,9 \%$ des sortants, soit 283871 diplômés. À l'avenir, les prévisions de la DPD ${ }^{71}$ tablent grosso modo sur un maintion des sorties annuelles constatées, depuis 1996, autour de 280000 (150000 au sein du supérieur long et 130000 au sein du supérieur court), en s'appuyant d'ailleurs, avec l'arrivée de classes creuses ${ }^{72}$, sur la réussite de politiques volontaristes (élargissement du recrutement des premiers cycles, succès de la licence professionnelle). Bien que ces prévisions n'intègrent ni les reprises d'études ni la formation continue, les tensions, voire les pénuries à venir sur le "marché des diplômés " de l'enseignement supérieur sont donc assez inéluctables sans que cela signifie dans l'absolu une pénurie spécifique de « scientifiques».

69. Globalement un tiers des diplômés de l'enseignement supérieur est déclassé au premier emploi, souvent plus en termes de CSP que de rémunération d'ailleurs, cf. E. Nauze-Fichet et M. Tomasini, « Diplóme et insertion sur le marché du travail : approches socioprofessionnelle et salariale du déclassement », Economie et statistique, $n^{\circ} 354$, 2002, pp. 21-48.

70. C. Sauvageot, « Les besoins en recrutement de jeunes sortant du système éducatif d'ici 2010 », Education et formations, n62, janvier-mars 2002, pp. 91-97.

71. P. Poulet-Coulibando, "Les prévisions de sorties de formation initiale ", Education et Formation, ${ }^{\circ} 63$, avril-juin 2002, pp. 87-91.

72. Qui connaissent cependant une stabilisation voire une réduction de la durée des études qui, paradoxalement, augmente les flux de sorties dans un premier temps, à la différence de ce qui s'est produit au tournant des années 1980-1990 : classes pleines mais allongement de la durée des études. 
À noter, d'ailleurs, que les tensions sur la formation et le recrutement des cadres devraient en toute logique amplifier le recours à la formation continue et le besoin...de formateurs (et, éventuellement, le rôle de l'université en la matière).

\section{Les besoins dans le champ de l'enseignement pri- maire et secondaire, supérieur et de la recherche publique}

Globalement $42 \%$ des enseignants « de la maternelle à l'université " partiront en retraite au cours de la décennie 2000-201073 :

- $44 \%$ dans le primaire ;

- $35 \%$ dans le second degré ;

- $31 \%$ des enseignants-chercheurs ;

- $27 \%$ des chercheurs (du secteur public) ;

- $38 \%$ des personnels de recherche (ing. techniciens, etc.).

Pour autant, les besoins de recrutement varient selon que l'on privilégie ou non des scénarios à effectifs constants 74 et selon de complexes hypothèses de redéploiements disciplinaires (par exemple au profit des sciences du vivant ou encore des sciences et technologies de l'information et de la communication).

À titre d'aperçu, les départs en retraite annuels ${ }^{75}$ seraient de :

- 16400 pour les enseignants du premier degré de 2003 à 2010 ; - 17300 pour les enseignants du second degré (public) de 2004 à 2010 ,

- 1400 pour les enseignants-chercheurs de 2000 à 2004 puis 1800 de 2005 à $2012^{76}$,

- 340 pour les chercheurs (en EPST) de 2000 à 2004 puis 1000 de 2005 à $2012^{77}$.

Ainsi, sans même compter les ITARF (environ 1000 départs annuels), les ITA des EPST (environ 1000 départs annuels) ou

73. Chiffres concernant les titulaires devant élèves, " Projection du système éducatif à 10 ans ", Education et formations, $n^{\circ} 63$, avril-juin 2002.

74. Pour le second degré public le besoin annuel de recrutement est d'environ 19000 contre 16900 en tenant compte des réductions d'effectifs scolaires.

75 « Projection du système éducatif à 10 ans », Education et formations, $n^{\circ} 63$, avril-juin 2002, op. cit.

76. R. Barré, M. Crance, A. Sigogneau, La recherche scientifique française : les enseignants-chercheurs et les chercheurs des EPST, situation démographique et perspective des départs 2001-2012, OST, avril 2002, 51 p. Ces départs concernent les plus de 65 ans ainsi qu'une proportion (déduite des comportements antérieurs, du taux de féminisation, etc.) des 60/64 ans.

77. Ibid. 
encore les effectifs des EPIC, les besoins pour assurer le seul renouvellement générationnel des enseignants (premier et second degré), enseignants-chercheurs et chercheurs publics seraient donc de l'ordre de 36000 diplômés par an (soit près de $12 \%$ des flux de sorties à $\mathrm{Bac}+2$ et $\mathrm{Bac}+3$ et plus aujourd'hui projetés à l'horizon 2010).

Dans les seuls champs « scientifiques » (selon une acception large intégrant certaines disciplines de l'enseignement technique et professionnel) et en excluant les enseignants du premier degré78, les besoins annuels moyens de recrutement pour les enseignants (second degré public), enseignants-chercheurs publics et chercheurs des EPST ${ }^{79}$ seraient entre 8500 et 11500 diplômés par an (des licenciés aux docteurs d'université) ${ }^{80}$.

En effet,

- les différents scénarios du ministère de l'Éducation nationale prévoient des besoins annuels de recrutement entre 7200 et 9800 pour les enseignants du second degré81 ;

- les travaux de l'OST estiment quant à eux les départs annuels d'enseignants-chercheurs universitaires et de chercheurs EPST entre 1200 et 1600 de 2001 à 2004 puis entre 1500 et 1900 entre 2005 et $2010^{82}$; soit un total entre 2003 et 2010 oscillant entre 69000 et 93000 diplômés.

En soulignant que ces besoins de recrutement (hors redéploiement) varient très fortement selon les disciplines (comme l'illustrent les annexes 15 et 16 du présent rapport).

Ces projections st calculs - présentés ici grossièrement peuvent être rapprochés des inscriptions en IUFM, aux concours de recrutement ou encore en thèse, à des fins d'illustration (et non de démarche projective).

78. Que l'on ne peut distinguer selon les disciplines..

79. Donc à l'exclusion des EPIC.

80. Pour une estimation des départs en retraite par discipline des enseignants du second degré cf. l'annexe $n^{\circ} 16$ et pour les enseignants-chercheurs universitaires l'annexe $n^{\circ} 17$. 81. D. Aussant, B. Lepetit, " Les besoins en personnels d'enseignement... ", Education et formations, $n^{\circ} 63$, avril-juin 2002 , op. cit. Soit dans le détail : entre $4200 / 5400$ en sciences, $1100 / 1500$ en génie mécanique et industriel, $30 / 1400$ en génie électrique, 100/250 en génie civil, 800/970 en économie et gestion, 600/800 en technologie.

82. R. Barré, M. Crance, A. Sigogneau, La recherche scientifique française, op. cit. Les fourchettes correspondent alu différentiel entre départs en retraite et départs totaux (comprenant les mobilités professionnelles), projetés à partir des comportements constatés dans la décennie 1990. 
À cet égard, trois séries de remarques.

Tout d'abord, dans un contexte où les besoins vont à nouveau croître, on ne peut qu'insister sur la très forte baisse des inscrits (comme des présents) aux concours externes (agrégations, CAPES, etc.) des disciplines scientifiques. Cette baisse est beaucoup plus significative que dans les disciplines de sciences humaines et de sciences sociales. De surcroît, il s'agit d'une baisse absolue alors même que le nombre de postes proposés ne cesse d'augmenter (pour les CAPES et CAPET) ${ }^{83}$.

De multiples raisons sont susceptibles d'expliquer cette réelle " désaffection " pour le coup à l'égard de ces concours externes des disciplines scientifiques ${ }^{84}$ du moins pour le second degré et le supérieur ${ }^{85}$, dont on soulignera surtout le fait qu'ils supposent désormais un cursus totalement distinct de celui de la recherche et, plus globalement, un cursus sans autres débouchés ${ }^{86}$.

Cette désaffection n'est qu'en partie compensée par la voie de fait que constitue la titularisation, plus ou moins récurrente, des personnels vacataires, intégrés après quelques années d'enseignement mais sans être soumis au mouvement national ${ }^{87}$.

Ensuite, quoique les besoins de recrutement des chercheurs et enseignants chercheurs ne représentent pas le même ordre de grandeur, les baisses d'inscriptions en doctorat (en dépit même du développement des inscriptions en DESS mais également en DEA, comme évoqué précédemment) laissent présager de fortes difficultés également dans ce champ ${ }^{88}$.

Sachant que les ratios actuels du nombre de docteurs qui deviennent in fine maîtres de conférence sont de l'ordre de $12 \%$ selon le rapport de l'IGAENR consacré au renouvellement des enseignants chercheurs ${ }^{89}$, en raison des précédentes décennies de

83. Cf. pour une présentation détaillée des postes, admis, inscrits et présents aux différents concours externes depuis 1999 , annexe $n^{\circ} 19$.

84. Qu'il serait cependant nécessaire de confronter à l'évolution des concours internes. 85. A la différence de la faveur qui touche le professorat des écoles.

86. A ce propos, on évoquera la difficulté - dans le cadre de cette mission - à connaître sur plusieurs années différentes données relatives aux IUFM. On soulignera juste, par exemple, que dans le cas de l'IdF et pour l'annee 2002, les inscrits en IUFM représentent $43,1 \%$ des inscrits au CAPES de mathématiques, $37,9 \%$ de ceux du capes de physique, $31,6 \%$ de ceux du CAPES sciences de la vie et de la Terre, $29,7 \%$ de ceux du CAPET génie civil, $30 \%$ de ceux du CAPET de mécanique, $17 \%$ de ceux du CAPET d'électronique, $36 \%$ de ceux du CAPET bio-sciences de la vie.

87. Près de 14000 postes ont ainsi été ouverts en 1998, contre 7000 en 2004.

88. Cf. annexe $n^{\circ} 18$.

89. M. Héon, Enseignants-chercheurs : le renouvellement des générations, rapport IGAENR, nov 2002. 
classes " pleines » ayant favorisé un système extrêmement sélectif. Or, même si une telle situation devra nécessairement évoluer en raison du " passage d'un état d'abondance à un état de rareté " (M. Héon $)^{90}$, le trend actuel des inscriptions en thèse pourrait s'avérer insuffisant pour assurer le renouvellement générationnel nécessaire.

Ainsi, même si le caractère actuellement aléatoire (ou plutôt très limité) d'accessibilité à un poste de chercheur ou d'enseignant chercheur devrait diminuer et donc s'avérer moins dissuasif, la préparation de la thèse demeure en l'état organisée « en tunnel », sans sortie « latérale » aisément envisageable.

Enfin, la plupart des travaux précédents ont insisté sur l'un des « viviers " prioritaires à développer, à savoir le renforcement de la féminisation, au sein des disciplines scientifiques, traditionnellement majoritairement masculines.

Sur ce point, on soulignera cependant, comme l'indique le tableau ci-dessous concernant des données franciliennes, que les sciences connaissent effectivement un taux de féminisation moindre que toutes les autres disciplines (et que de surcroît il tendrait à baisser légèrement entre 1996 et 2002 !). Toutefois, on remarquera également que le « décrochage » entre $2^{\mathrm{e}}$ et $3^{\mathrm{e}}$ cycles est moins prononcé dans le cas des sciences que dans les secteurs non scientifiques. Ainsi, quoique moins présentes, les étudiantes de sciences auraient tendance néanmoins - en part relative - à mieux se maintenir en troisième cycle. Les possibilités de réserve en seraient donc peut-être plus réduites ${ }^{91}$.

90. Ibid.

91. Mais ceci demeure encore une piste à explorer, en intégrant notamment les enjeux de taux de féminisation au sein des écoles, et plus largement des filières non universitaires... 


\begin{tabular}{|c|c|c|c|c|c|c|c|c|}
\hline \multirow{3}{*}{ Disciplines } & \multicolumn{8}{|c|}{ Inscriptions en $Z$ et $3^{\prime}$ cycles en IdF : Taux de féminisation 1996-2002 } \\
\hline & \multicolumn{4}{|c|}{1996} & \multicolumn{4}{|c|}{2002} \\
\hline & \multicolumn{2}{|c|}{$2^{\epsilon}$ cycle } & \multicolumn{2}{|c|}{$3^{\circ}$ cycle } & \multicolumn{2}{|c|}{$2^{e}$ cycle } & \multicolumn{2}{|c|}{$3^{\circ}$ cycle } \\
\hline Médicales & $4888 / 9952$ & $49,1 \%$ & $9999 / 21025$ & $47,2 \%$ & $4194 / 7108$ & $59 \%$ & $11439 / 22140$ & $51,7 \%$ \\
\hline Scientifiques & $7974 / 19943$ & $40 \%$ & $5550 / 14426$ & $38,5 \%$ & $8338 / 21406$ & $39 \%$ & $5333 / 14088$ & $37,9 \%$ \\
\hline Méd + scien & $12862 / 29895$ & $43 \%$ & $15549 / 35451$ & $43,9 \%$ & $12532 / 28514$ & $44 \%$ & $16772 / 36228$ & $46,3 \%$ \\
\hline Non scientif & $65493 / 99361$ & $65,9 \%$ & $23359 / 40250$ & $58 \%$ & $67437 / 102832$ & $65,6 \%$ & $25843 / 43793$ & $59 \%$ \\
\hline Ttes discipl. & $78355 / 129256$ & $60,6 \%$ & $38908 / 75701$ & $51,4 \%$ & $79969 / 131346$ & $60,9 \%$ & $42615 / 80021$ & $53,3 \%$ \\
\hline
\end{tabular}

Source : BCP, SISE 
Ainsi, la décrue affectant actuellement les DEUG scientifiques constitue donc surtout un problème d'abord pour le recrutement des enseignants du premier et du second degré, puis pour celui des enseignants-chercheurs et des chercheurs si cette décrue du premier cycle n'est pas compensée en deuxième et troisième cycle par des flux en provenance d'autres filières. D'autant que ces recrutements seront bien confrontés à une concurrence amplifiée de ceux nécessaires à la recherche privée (à la démographie plus jeune mais susceptible de connaître de fortes créations d'emploi) ${ }^{92}$, au secteur informatique ${ }^{93}$, aux ingénieurs de l'industrie, des cadres technico-commerciaux, etc., sachant que, dans certains de ces secteurs, seul le vivier scolaire (et non les possibilités de promotion interne) est susceptible de fournir les nouveaux cadres nécessaires.

92. Le chiffre de recrutement net (renouvellement face aux retraites et création d'emplois) avancés par la DARES, soit $\mathbf{2 3 0} 000$ postes dans la décennie, pour les « chercheurs " correspond vraisemblablement à l'ensemble du secteur recherche (publique et privée, chercheurs, ingénieurs, cadres de recherche et non cadres).

93. Soit 250000 emplois sur la décennie, selon la DARES. 